\title{
Fixed point theorems on cone 2-metric spaces over Banach algebras and an application
}

\section{Tao Wang, Jiandong Yin * and Qi Yan}

"Correspondence: yjdaxf@163.com Department of Mathematics, Nanchang University, Nanchang, 330031, P.R. China

\begin{abstract}
In this paper, the concept of cone 2-metric spaces over Banach algebras is introduced and some existence and uniqueness theorems of fixed points for some mappings satisfying certain contractive conditions are established. As an application of one of the main results, an example is given at the end of the paper.
\end{abstract}

MSC: $54 \mathrm{H} 25 ; 47 \mathrm{H} 40$

Keywords: cone 2-metric spaces over Banach algebras; fixed point theorems; spectral radius

\section{Introduction}

The notion of 2-metric spaces can be dated back to the work of Gähler, who established some fixed point theorems in 2-metric spaces in [1-3]. In 1976, Sharma et al. in [4] investigated the existence and uniqueness of the fixed points of a family of mappings in 2-metric spaces as follows.

Theorem 1.1 Let $(X, d)$ be a complete bounded 2-metric space (namely, there exists a constant $K$ such that $d(a, b, c) \leq K$ for all $a, b, c \in X)$ and let $\left\{T_{i}\right\}_{i=1}^{\infty}$ be a family of mappings from $X$ to itself. Suppose that there exists a non-negative integer sequence $\left\{m_{i}\right\}_{i=1}^{\infty}$ such that for all positive integers $i, j$ and for all $x, y, a \in X$, we have

$$
d\left(T_{i}^{m_{i}} x, T_{j}^{m_{j}} y, a\right) \leq \alpha\left(d\left(x, T_{i}^{m_{i}} x, a\right)+d\left(y, T_{j}^{m_{j}} y, a\right)\right)+\beta d(x, y, a)
$$

for non-negative constants $\alpha, \beta$ with $2 \alpha+\beta<1$. Then $\left\{T_{i}\right\}$ share a unique fixed point in $X$.

In 1979, Rhoades [5] obtained the following.

Theorem 1.2 Let $(X, d)$ be a complete 2-metric space. Suppose the mapping $T: X \rightarrow X$ satisfies

$$
d(T x, T y, a) \leq h \max \{d(x, y, a), d(x, T x, a), d(y, T y, a), d(x, T y, a), d(y, T x, a)\}
$$

for $0 \leq h<1$. Then $T$ has a unique fixed point $x$ in $X$ and for every $x_{0} \in X$, the iterative sequence $\left\{T^{n} x_{0}\right\}$ converges to $x$. Besides, the following error estimate formula

(c) 2015 Wang et al. This article is distributed under the terms of the Creative Commons Attribution 4.0 International License (http://creativecommons.org/licenses/by/4.0/), which permits unrestricted use, distribution, and reproduction in any medium, provided you give appropriate credit to the original author(s) and the source, provide a link to the Creative Commons license, and indicate if changes were made. 
holds:

$$
d\left(T^{n} x_{0}, x, a\right) \leq \frac{h^{n}}{1-h} d\left(x_{0}, T x_{0}, a\right) .
$$

In 2007, by defining the distance $d(x, y)$ as a vector in an ordered Banach space $E$, Huang and Zhang [6] introduced the concept of cone metric spaces and gave the new version of the Banach contraction principle in the setting of cone metric spaces. Since then, a few fixed point results have been proven on cone metric spaces (see [7-11] for more details). Moreover, Liu and $\mathrm{Xu}$ [12] introduced the concept of cone metric spaces over Banach algebras and showed that cone metric spaces over Banach algebras are not equivalent to metric spaces. Based on the work of Liu and Xu [12], the authors of [13, 14] obtained some interesting results on cone metric spaces over Banach algebras. By combining the concepts of 2-metric spaces and cone metric spaces, Singh et al. [15] introduced a new space, which is called a cone 2-metric space and established some fixed point theorems of a contractive mapping on cone 2-metric spaces.

Enlightened by these thoughts, we define a new space in this paper, which is called the cone 2-metric space over a Banach algebra and prove some fixed point theorems of some mappings satisfying certain contractive conditions. Our main results extend Theorem 1.1 and Theorem 1.2 to cone 2-metric spaces over Banach algebras. Moreover, some frequently used conditions as the normality of a cone and the boundedness of a metric space are not needed in our results. In addition, the proofs of our results are quite different from those of some known results since a given pair of elements in a Banach algebra may not be compared.

\section{Preliminaries}

Let $\mathcal{A}$ always be a real Banach algebra, that is, $\mathcal{A}$ is a real Banach space in which an operation of multiplication is defined, subject to the following properties (for all $x, y, z \in \mathcal{A}$, $a \in \mathbb{R}):$

(i) $(x y) z=x(y z)$;

(ii) $x(y+z)=x y+x z$ and $(x+y) z=x z+y z$;

(iii) $\alpha(x y)=(\alpha x) y=x(\alpha y)$;

(iv) $\|x y\| \leq\|x\|\|y\|$.

Throughout this paper, we shall assume that a Banach algebra has a unit (i.e., a multiplicative identity) $e$ such that $e x=x e=x$ for all $x \in \mathcal{A}$. An element $x \in \mathcal{A}$ is said to be invertible if there is an inverse element $y \in \mathcal{A}$ such that $x y=y x=e$. The inverse of $x$ is denoted by $x^{-1}$. For more details, we refer to [16].

The following several lemmas about spectral radius are needed in this paper.

Lemma 2.1 (see [16]) Let $\mathcal{A}$ be a Banach algebra with a unit e and $x \in \mathcal{A}$. If the spectral radius $r(x)$ of $x$ is less than 1 , i.e.,

$$
r(x)=\lim _{n \rightarrow \infty}\left\|x^{n}\right\|^{\frac{1}{n}}=\inf _{n \geq 1}\left\|x^{n}\right\|^{\frac{1}{n}}<1,
$$

then $e-x$ is invertible. Actually

$$
(e-x)^{-1}=\sum_{i=0}^{\infty} x^{i}
$$


Remark 2.1 If $r(x)<1$, then $\left\|x^{n}\right\| \rightarrow 0(n \rightarrow \infty)$.

Lemma 2.2 (see [17]) Let $\mathcal{A}$ be a Banach algebra and let $x, y$ be vectors in $\mathcal{A}$. If $x$ and $y$ commute, then the following hold:

(i) $r(x y) \leq r(x) r(y)$;

(ii) $r(x+y) \leq r(x)+r(y)$;

(iii) $|r(x)-r(y)| \leq r(x-y)$.

Lemma 2.3 (see [17]) Let $\mathcal{A}$ be a Banach algebra and let $k$ be a vector in $\mathcal{A}$. If $0 \leq r(k)<1$, then we have

$$
r\left((e-k)^{-1}\right) \leq(1-r(k))^{-1}
$$

Now let us recall the concepts of cone and partial ordering for the Banach algebra $\mathcal{A}$. A subset $P$ of $\mathcal{A}$ is called a cone of $\mathcal{A}$ if:

(i) $P$ is non-empty closed and $\{\theta, e\} \subset P$;

(ii) $\alpha P+\beta P \subset P$ for all non-negative real numbers $\alpha, \beta$;

(iii) $P^{2}=P P \subset P$;

(iv) $P \cap(-P)=\{\theta\}$,

where $\theta$ denotes the null of the Banach algebra $\mathcal{A}$. For a given cone $P \subset \mathcal{A}$, we can define a partial ordering $\preceq$ with respect to $P$ by $x \preceq y$ if and only if $y-x \in P . x \prec y$ will stand for $x \preceq y$ and $x \neq y$, while $x \ll y$ will stand for $y-x \in \operatorname{int} P$, where int $P$ denotes the interior of $P$. If int $P \neq \emptyset$, then $P$ is called a solid cone.

In the following we always assume that $P$ is a cone in $\mathcal{A}$ with int $P \neq \emptyset$ and $\preceq$ is the partial ordering with respect to $P$.

Definition 2.1 (see [1]) Let $X$ be a non-empty set. Suppose that the mapping $d: X \times X \times$ $X \rightarrow \mathbb{R}^{+}$satisfies:

(i) for every pair of distinct points $x, y \in X$, there exists a point $z \in X$ such that

$$
d(x, y, z) \neq 0
$$

(ii) $d(x, y, z)=0$ if and only if at least two of $x, y, z$ are equal;

(iii) $d(x, y, z)=d(p(x, y, z))$ for all $x, y, z \in X$ and for all permutations $p(x, y, z)$ of $x, y, z$;

(iv) $d(x, y, z) \leq d(x, y, w)+d(x, w, z)+d(w, y, z)$ for all $x, y, z, w \in X$.

Then $d$ is called a 2-metric on $X$, and $(X, d)$ is called a 2-metric space.

Definition 2.2 Let $X$ be a non-empty set. Suppose that the mapping $d: X \times X \times X \rightarrow \mathcal{A}$ satisfies:

(i) for every pair of distinct points $x, y \in X$, there exists a point $z \in X$ such that

$$
d(x, y, z) \neq \theta \text {; }
$$

(ii) $\theta \preceq d(x, y, z)$ for all $x, y, z \in X$ and $d(x, y, z)=\theta$ if and only if at least two of $x, y, z$ are equal;

(iii) $d(x, y, z)=d(p(x, y, z))$ for all $x, y, z \in X$ and for all permutations $p(x, y, z)$ of $x, y, z$;

(iv) $d(x, y, z) \preceq d(x, y, w)+d(x, w, z)+d(w, y, z)$ for all $x, y, z, w \in X$.

Then $d$ is called a cone 2-metric on $X$, and $(X, d)$ is called a cone 2-metric space over the Banach algebra $\mathcal{A}$.

See [15] for some special examples of cone 2-metric spaces over Banach algebras. 
Definition 2.3 Let $(X, d)$ be a cone 2-metric space over the Banach algebra $\mathcal{A}, x \in X$, and let $\left\{x_{n}\right\}$ be a sequence in $X$. Then:

(i) $\left\{x_{n}\right\}$ converges to $x$ whenever for each $c \in \mathcal{A}$ with $\theta \ll c$ there is a natural number $N$ such that $d\left(x_{n}, x, a\right) \ll c$ for all $a \in X$ and for all $n \geq N$. We denote it by $\lim _{n \rightarrow \infty} x_{n}=x$ or $x_{n} \rightarrow x$ as $n \rightarrow \infty$.

(ii) $\left\{x_{n}\right\}$ is a Cauchy sequence whenever for each $c \in \mathcal{A}$ with $\theta \ll c$ there is a natural number $N$ such that $d\left(x_{n}, x_{m}, a\right) \ll c$ for all $a \in X$ and for all $n, m \geq N$.

(iii) $(X, d)$ is a complete cone 2-metric space if every Cauchy sequence is convergent in $X$.

Lemma 2.4 (see [18]) If $E$ is a real Banach space with a solid cone $P$ and if $\theta \preceq u \ll c$ for each $\theta \ll c$, then $u=\theta$.

Lemma 2.5 (see [18]) If $\mathcal{A}$ is a real Banach space with a solid cone $P$ and if $\left\|x_{n}\right\| \rightarrow 0$ $(n \rightarrow \infty)$, then for any $\theta \ll c$, there exists $N \in \mathbb{N}$ such that, for any $n>N$, we have $x_{n} \ll c$.

Now, we review some facts on $c$-sequence theories.

Definition 2.4 (see $[19,20]$ ) Let $P$ be a solid cone in a Banach space $\mathcal{A}$. A sequence $\left\{x_{n}\right\} \subset$ $P$ is a $c$-sequence if for each $\theta \ll c$ there exists $N \in \mathbb{N}$ such that $x_{n} \ll c$ for all $n>N$.

Lemma 2.6 (see [20]) Let $P$ be a solid cone in a Banach space $\mathcal{A}$ and let $\left\{x_{n}\right\}$ and $\left\{y_{n}\right\}$ be sequences in P. If $\left\{x_{n}\right\}$ and $\left\{y_{n}\right\}$ are c-sequences and $\alpha, \beta>0$, then $\left\{\alpha x_{n}+\beta y_{n}\right\}$ is a c-sequence.

Lemma 2.7 (see [17]) Let $P$ be a solid cone in a Banach space $\mathcal{A}$ and let $\left\{x_{n}\right\}$ be a sequence in $P$. Suppose that $k \in P$ is an arbitrarily given vector and $\left\{x_{n}\right\}$ is a c-sequence in $P$. Then $\left\{k x_{n}\right\}$ is a c-sequence.

\section{Main results and proofs}

In this section, we will give some fixed point theorems in the setting of cone 2-metric spaces over Banach algebras.

Proposition 3.1 Let $(X, d)$ be a complete cone 2-metric space over the Banach algebra $\mathcal{A}$ and let $P$ be the underlying solid cone in $\mathcal{A}$. Let $\left\{x_{n}\right\}$ be a sequence in $X$. If $\left\{x_{n}\right\}$ converges to $x \in X$, then we have:

(i) $\left\{d\left(x_{n}, x, a\right)\right\}$ is a c-sequence for all $a \in X$.

(ii) For any $p \in \mathbb{N},\left\{d\left(x_{n}, x_{n+p}, a\right)\right\}$ is a c-sequence for all $a \in X$.

Proof The proof of this proposition is easy, so we omit it here.

Proposition 3.2 Let $P$ be a solid cone in the Banach algebra $\mathcal{A}$ and let $x$ be a vector in $\mathcal{A}$. Suppose that $k \in P$ is an arbitrarily given vector and $x \ll c$ for any $\theta \ll c$, then we have $k x \ll c$ for any $\theta \ll c$.

Proof Fix $c \gg \theta$, then $\frac{c}{m} \gg \theta$ for all $m \in \mathbb{N}$. It is clear that $x \preceq \frac{c}{m}$ for all $m \in \mathbb{N}$. So $k x \preceq \frac{k c}{m}$. Since $\frac{k c}{m} \rightarrow \theta$ as $m \rightarrow \infty$, there exists $M \in \mathbb{N}$ such that $k x \preceq \frac{k c}{m} \ll c$ when $m>M$. This completes the proof. 
Theorem 3.1 Let $(X, d)$ be a complete cone 2-metric space over the Banach algebra $\mathcal{A}$ and $P$ be the underlying solid cone and $\left\{T_{i}\right\}_{i=1}^{\infty}$ be a family of mappings from $X$ to itself. Suppose that there exists a non-negative integer sequence $\left\{m_{i}\right\}_{i=1}^{\infty}$ such that for all positive integers $i$, $j$ and for all $x, y, a \in X$, we have

$$
d\left(T_{i}^{m_{i}} x, T_{j}^{m_{j}} y, a\right) \preceq k_{1} d\left(x, T_{i}^{m_{i}} x, a\right)+k_{2} d\left(y, T_{j}^{m_{j}} y, a\right)+k_{3} d(x, y, a)
$$

for $k_{1}, k_{2}, k_{3} \in P$ with $r\left(k_{1}\right)+r\left(k_{2}\right)+r\left(k_{3}\right)<1$ and $k_{1}, k_{2}$, and $k_{3}$ commute. Then $\left\{T_{i}\right\}_{i=1}^{\infty}$ share a unique fixed point in $X$.

Proof Write $g_{i}=T_{i}^{m_{i}}, i=1,2, \ldots$. Then for all $i, j$, by (3.1), we have

$$
d\left(g_{i}(x), g_{j}(y), a\right) \preceq k_{1} d\left(x, g_{i}(x), a\right)+k_{2} d\left(y, g_{j}(y), a\right)+k_{3} d(x, y, a) .
$$

Pick $x_{0} \in X$ and set $x_{n}=g_{n}\left(x_{n-1}\right), n \geq 1$. Then

$$
\begin{aligned}
d\left(x_{n+1}, x_{n}, a\right) & =d\left(g_{n+1}\left(x_{n}\right), g_{n}\left(x_{n-1}\right), a\right) \\
& \preceq k_{1} d\left(x_{n}, x_{n+1}, a\right)+k_{2} d\left(x_{n-1}, x_{n}, a\right)+k_{3} d\left(x_{n}, x_{n-1}, a\right),
\end{aligned}
$$

which together with Lemma 2.1 yields

$$
\begin{aligned}
d\left(x_{n+1}, x_{n}, a\right) \preceq & k d\left(x_{n}, x_{n-1}, a\right) \\
\preceq & k^{2} d\left(x_{n-1}, x_{n-2}, a\right) \\
& \vdots \\
\preceq & k^{n} d\left(x_{1}, x_{0}, a\right),
\end{aligned}
$$

where $k=\left(e-k_{1}\right)^{-1}\left(k_{2}+k_{3}\right)$. In this case, for all $l<n$, one has

$$
\begin{aligned}
d\left(x_{n}, x_{n-1}, x_{l}\right) \preceq & k d\left(x_{n-1}, x_{n-2}, x_{l}\right) \\
& \vdots \\
\preceq & k^{n-l-1} d\left(x_{l+1}, x_{l}, x_{l}\right) .
\end{aligned}
$$

It means that $d\left(x_{n}, x_{n-1}, x_{l}\right)=\theta$ for all $l<n$. Thus, for $n>m$, we have

$$
\begin{aligned}
d\left(x_{n}, x_{m}, a\right) \preceq & d\left(x_{n}, x_{m}, x_{n-1}\right)+d\left(x_{n}, x_{n-1}, a\right)+d\left(x_{n-1}, x_{m}, a\right) \\
\preceq & k^{n-1} d\left(x_{1}, x_{0}, a\right)+d\left(x_{n-1}, x_{m}, x_{n-2}\right)+d\left(x_{n-1}, x_{n-2}, a\right)+d\left(x_{n-2}, x_{m}, a\right) \\
\preceq & \left(k^{n-1}+k^{n-2}\right) d\left(x_{1}, x_{0}, a\right)+d\left(x_{n-2}, x_{m}, a\right) \\
& \vdots \\
\preceq & \left(k^{n-1}+k^{n-2}+\cdots+k^{m+1}\right) d\left(x_{1}, x_{0}, a\right)+d\left(x_{m+1}, x_{m}, a\right) \\
\preceq & \left(k^{n-1}+k^{n-2}+\cdots+k^{m+1}+k^{m}\right) d\left(x_{1}, x_{0}, a\right)
\end{aligned}
$$




$$
\begin{aligned}
& =\left(e+k+\cdots+k^{n-m+1}\right) k^{m} d\left(x_{1}, x_{0}, a\right) \\
& \preceq\left(\sum_{i=0}^{\infty} k^{i}\right) k^{m} d\left(x_{1}, x_{0}, a\right) \\
& =(e-k)^{-1} k^{m} d\left(x_{1}, x_{0}, a\right) .
\end{aligned}
$$

On the other hand, it follows from Lemma 2.2 and Lemma 2.3 that

$$
\begin{aligned}
r(k)=r\left(e-k_{1}\right)^{-1}\left(k_{2}+k_{3}\right) & \leq r\left(\left(e-k_{1}\right)^{-1}\right) r\left(k_{2}+k_{3}\right) \\
& \leq \frac{r\left(k_{2}+k_{3}\right)}{1-r\left(k_{1}\right)} \\
& \leq \frac{r\left(k_{2}\right)+r\left(k_{3}\right)}{1-r\left(k_{1}\right)}<1 .
\end{aligned}
$$

Hence, from Lemma 2.5 and the fact that $\left\|(e-k)^{-1} k^{m} d\left(x_{1}, x_{0}, a\right)\right\| \rightarrow 0(n \rightarrow \infty)$, it follows that, for any $c \in \mathcal{A}$ with $\theta \ll c$, there exists $N \in \mathbb{N}$ such that for any $n>m>N$, we have

$$
d\left(x_{n}, x_{m}, a\right) \preceq(e-k)^{-1} k^{m} d\left(x_{1}, x_{0}, a\right) \ll c .
$$

So $\left\{x_{n}\right\}$ is a Cauchy sequence in $X$. As $X$ is complete, there exists $x \in X$ such that $x_{n} \rightarrow x$ $(n \rightarrow \infty)$. We claim that $x$ is the unique fixed point of $T_{i}$ for all $i \geq 1$. In fact

$$
\begin{aligned}
d\left(g_{n}(x), x, a\right) \preceq & d\left(g_{n}(x), x, g_{m+1}\left(x_{m}\right)\right)+d\left(g_{n}(x), g_{m+1}\left(x_{m}\right), a\right)+d\left(g_{m+1}\left(x_{m}\right), x, a\right) \\
\preceq & d\left(x_{m+1}, x, a\right)+k_{1} d\left(x, g_{n}(x), x\right)+k_{2} d\left(x_{m}, g_{m+1}\left(x_{m}\right), x\right)+k_{3} d\left(x, x_{m}, x\right) \\
& +k_{1} d\left(x_{m}, g_{m+1}\left(x_{m}\right), a\right)+k_{2} d\left(x, g_{n}(x), a\right)+k_{3} d\left(x_{m}, x, a\right),
\end{aligned}
$$

which implies

$$
\left(e-k_{2}\right) d\left(g_{n}(x), x, a\right) \preceq d\left(x_{m+1}, x, a\right)+k_{2} d\left(x_{m}, x_{m+1}, x\right)+k_{1} d\left(x_{m}, x_{m+1}, a\right)+k_{3} d\left(x_{m}, x, a\right) .
$$

Therefore, it follows from Lemma 2.6, Lemma 2.7, and Proposition 3.1 that $\left(e-k_{2}\right) d\left(g_{n}(x)\right.$, $x, a) \preceq y_{m}$, where $\left\{y_{m}\right\}$ is a $c$-sequence in $P$. In this case, we have $\left(e-k_{2}\right) d\left(g_{n}(x), x, a\right) \ll c$ for any $c \gg \theta$, which together with Proposition 3.2 implies that $\theta \preceq d\left(g_{n}(x), x, a\right) \ll c$ for any $a \in X, n \in \mathbb{N}$, and $c \gg \theta$ as $e-k_{2}$ is invertible. Therefore, by Lemma $2.4, d\left(g_{n}(x), x, a\right)=\theta$ for any $n \in \mathbb{N}$. Namely, $g_{n}(x)=x$ for any $n \in \mathbb{N}$.

Suppose that $y$ is another fixed point of $g_{n}$ in $X$, then

$$
d(x, y, a) \preceq k_{1} d(x, x, a)+k_{2} d(y, y, a)+k_{3} d(x, y, a) .
$$

That is $\left(e-k_{3}\right) d(x, y, a) \preceq \theta$, which means $d(x, y, a)=\theta$ for any $a \in X$ because of the invertibility of $e-k_{3}$. So $x=y$. That is, the fixed point of $g_{n}$ is unique. As $x=g_{n}(x)=T_{n}^{m_{n}}(x)$, we have $T_{n}(x)=T_{n}\left(T_{n}^{m_{n}}(x)\right)=T_{n}^{m_{n}}\left(T_{n}(x)\right)=g_{n}\left(T_{n}(x)\right)$. It is easy to see that $T_{n}(x)=x$. Similarly, the uniqueness of the fixed point of $T_{n}$ can be proven.

Remark 3.1 If we replace the Banach algebra $\mathcal{A}$ with the real numbers field $\mathbb{R}$ in Theorem 3.1, then we get a similar result as Theorem 1.1. Moreover, it should be noted that, in 
this case, we do not need the bounded property of the underlying space $(X, d)$, so Theorem 3.1 generalizes and extends Theorem 1.1.

Corollary 3.1 Let $(X, d)$ be a complete cone 2-metric space over the Banach algebra $\mathcal{A}$ and $P$ be the underlying solid cone and let $\left\{T_{i}\right\}_{i=1}^{\infty}$ be a family of mappings from $X$ to itself. Suppose that there exists a non-negative integer sequence $\left\{m_{i}\right\}_{i=1}^{\infty}$ such that for all positive integers $i, j$ and for all $x, y, a \in X$, we have

$$
d\left(T_{i}^{m_{i}} x, T_{j}^{m_{j}} y, a\right) \preceq k d(x, y, a)
$$

for some fixed $k \in P$ with $r(k)<1$. Then the $\left\{T_{i}\right\}$ share a unique fixed point in $X$.

Corollary 3.2 Let $(X, d)$ be a complete cone 2-metric space over the Banach algebra $\mathcal{A}$ and $P$ be the underlying solid cone and let $\left\{T_{i}\right\}_{i=1}^{\infty}$ be a family of mappings from $X$ to itself. Suppose that there exists a non-negative integer sequence $\left\{m_{i}\right\}_{i=1}^{\infty}$ such that for all positive integers $i, j$ and for all $x, y, a \in X$, we have

$$
d\left(T_{i}^{m_{i}} x, T_{j}^{m_{j}} y, a\right) \preceq k\left(d\left(x, T_{i}^{m_{i}} x, a\right)+d\left(y, T_{j}^{m_{j}} y, a\right)\right)
$$

for some fixed $k \in P$ with $r(k)<\frac{1}{2}$. Then the $\left\{T_{i}\right\}$ share a unique fixed point in $X$.

Proof Note that $r(k+k) \leq 2 r(k)<1$ according to Lemma 2.2, so the proof is rightward from Theorem 3.1.

In the following, we will present some notations. Let $a_{i}, b_{i}, x, y, k \in \mathcal{A}$. Write:

(i) $x \preceq \bigvee_{i \geq 0}\left\{a_{i}\right\} \Leftrightarrow$ there exists $i \geq 0$ such that $x \preceq a_{i}$.

(ii) $\bigvee_{i \geq 0}\left\{a_{i}\right\} \preceq \bigvee_{j \geq 0}\left\{b_{j}\right\} \Leftrightarrow$ for any $i \geq 0$ there exists $j \geq 0$ such that $a_{i} \preceq b_{j}$.

(iii) $\bigvee_{i \geq 0}\left\{a_{i}\right\} \preceq x \Leftrightarrow$ for any $i \geq 0$, we have $a_{i} \preceq x$.

(iv) $k \bigvee_{i \geq 0}\left\{a_{i}\right\}=\bigvee_{i \geq 0}\left\{k a_{i}\right\}$

Lemma 3.1 (see [17]) Let $\mathcal{A}$ be a Banach algebra with a unit e, $P$ be a cone in $\mathcal{A}$, and $\preceq$ be the partial ordering generated by $P$. Suppose that $x$ is invertible and that $x^{-1} \succ \theta$ implies $x \succ \theta$. Let $\lambda \in P$. If the spectral radius $r(\lambda)$ of $\lambda$ is less than 1 , then for any integer $n \geq 1$, we have $\lambda^{n} \preceq \lambda \preceq e$.

Lemma 3.2 Let $(X, d)$ be a complete cone 2-metric space, $P$ be a cone in the Banach algebra $\mathcal{A}$ and $\leq$ be the partial ordering generated by P. Let $\left\{x_{n}\right\}$ and $\left\{y_{n}\right\}$ be two sequences in $X$ with $\lim _{n \rightarrow \infty} x_{n}=x$ and $\lim _{n \rightarrow \infty} y_{n}=y$. Then for any $c \gg \theta$, there exists $N \in \mathbb{N}$ such that for any $n>N$, we have

$$
-c \preceq d\left(x_{n}, y_{n}, a\right)-d(x, y, a) \preceq c
$$

for all $a \in X$.

Proof The proof is easy, so we omit it here.

Lemma 3.3 Let $(X, d)$ be a complete cone 2-metric space over the Banach algebra $\mathcal{A}$ and $P$ be the underlying solid cone. 
(1) Suppose that $x$ is invertible and that $x^{-1} \succ \theta$ implies $x \succ \theta$.

(2) Suppose the mapping $T: X \rightarrow X$ satisfies

$$
d(T x, T y, a) \preceq k \bigvee\{d(x, y, a), d(x, T x, a), d(y, T y, a) d(x, T y, a), d(y, T x, a)\}
$$

for an invertible $k \in P$ with $r(k)<1$.

Then for any positive integers $n$ and any $x_{0} \in X$, the following assertions hold:

(i) $\bigvee_{1 \leq i, j \leq n}\left\{d\left(T^{i} x_{0}, T^{j} x_{0}, a\right)\right\} \preceq k \bigvee_{0 \leq i, j \leq n}\left\{d\left(T^{i} x_{0}, T^{j} x_{0}, a\right)\right\}$.

(ii) $\bigvee_{1 \leq i, j \leq n}\left\{d\left(T^{i} x_{0}, T^{j} x_{0}, a\right)\right\} \preceq k \bigvee_{0 \leq j \leq n}\left\{d\left(x_{0}, T^{j} x_{0}, a\right)\right\}$.

(iii) For all $i, j=1,2, \ldots, n$, we have $d\left(T^{i} x_{0}, T^{j} x_{0}, x_{0}\right)=\theta$.

(iv) For any $n \in \mathbb{N}$,

$$
\bigvee_{0 \leq i, j \leq n}\left\{d\left(T^{i} x_{0}, T^{j} x_{0}, a\right)\right\} \preceq\left((e-k)^{-1}(e-k)^{-1}+(e-k)^{-1}\right) d\left(x_{0}, T x_{0}, a\right)
$$

Proof According to (3.2), for all $i, j, 1 \leq i, j \leq n$, we have

$$
\begin{aligned}
d\left(T^{i} x_{0}, T^{j} x_{0}, a\right)= & d\left(T T^{i-1} x_{0}, T T^{j-1} x_{0}, a\right) \\
\preceq & k \bigvee\left\{d\left(T^{i-1} x_{0}, T^{j-1} x_{0}, a\right), d\left(T^{i-1} x_{0}, T^{i} x_{0}, a\right), d\left(T^{j-1} x_{0}, T^{j} x_{0}, a\right),\right. \\
& \left.d\left(T^{i-1} x_{0}, T^{j} x_{0}, a\right), d\left(T^{j-1} x_{0}, T^{i} x_{0}, a\right)\right\} \\
\preceq & k \bigvee_{0 \leq i, j \leq n}\left\{d\left(T^{i} x_{0}, T^{j} x_{0}, a\right)\right\} .
\end{aligned}
$$

So (i) is true.

From (i), we know that

$$
\bigvee_{1 \leq i, j \leq n}\left\{d\left(T^{i} x_{0}, T^{j} x_{0}, a\right)\right\} \preceq k \bigvee\left\{\bigvee_{1 \leq i, j \leq n}\left\{d\left(T^{i} x_{0}, T^{j} x_{0}, a\right)\right\}, \bigvee_{0 \leq j \leq n}\left\{d\left(x_{0}, T^{j} x_{0}, a\right)\right\}\right\}
$$

We claim that $\bigvee_{1 \leq i, j \leq n}\left\{d\left(T^{i} x_{0}, T^{j} x_{0}, a\right)\right\} \preceq k \bigvee_{0 \leq j \leq n}\left\{d\left(x_{0}, T^{j} x_{0}, a\right)\right\}$. In fact, for any $1 \leq i, j \leq$ $n$, there exist $1 \leq i^{(1)}, j^{(1)} \leq n$ such that $d\left(T^{i} x_{0}, T^{j} x_{0}, a\right) \preceq k d\left(T^{i^{(1)}} x_{0}, T^{j^{(1)}} x_{0}, a\right)$ (otherwise $d\left(T^{i} x_{0}, T^{j} x_{0}, a\right) \preceq k d\left(x_{0}, T^{j_{1}} x_{0}, a\right)$ for some $0 \leq j_{1} \leq n$, then the claim is true). Also, there exist $1 \leq i^{(2)}, j^{(2)} \leq n$ such that $d\left(T^{i^{(1)}} x_{0}, T^{j^{(1)}} x_{0}, a\right) \preceq k d\left(T^{i^{(2)}} x_{0}, T^{j^{(2)}} x_{0}, a\right)$ (otherwise, by Lemma 3.1, $d\left(T^{i} x_{0}, T^{j} x_{0}, a\right) \preceq k\left(k d\left(x_{0}, T^{j_{2}} x_{0}, a\right)\right)=k^{2} d\left(x_{0}, T^{j_{2}} x_{0}, a\right) \preceq k d\left(x_{0}, T^{j_{2}} x_{0}, a\right)$ for some $0 \leq j_{2} \leq n$; so the claim is true). As the procedure continues, we obtain

$$
\begin{aligned}
d\left(T^{i} x_{0}, T^{j} x_{0}, a\right) \preceq & k d\left(T^{i^{(1)}} x_{0}, T^{j^{(1)}} x_{0}, a\right) \\
\preceq & k^{2} d\left(T^{i^{(2)}} x_{0}, T^{j^{(2)}} x_{0}, a\right) \\
& \vdots \\
\preceq & k^{t} d\left(T^{i^{(t)}} x_{0}, T^{j^{(t)}} x_{0}, a\right) .
\end{aligned}
$$

Otherwise, the claim is true. Besides, there exists $0 \leq s<t$ such that $i^{(s)}=i^{(t)}, j^{(s)}=$ $j^{(t)}$, where $i^{(0)}=i, j^{(0)}=j$. Then we have $k^{s} d\left(T^{i^{(s)}} x_{0}, T^{j^{(s)}} x_{0}, a\right) \preceq k^{t} d\left(T^{i^{(t)}} x_{0}, T^{j^{(t)}} x_{0}, a\right)=$ 
$k^{t} d\left(T^{i^{(s)}} x_{0}, T^{(s)} x_{0}, a\right)$, which implies

$$
\left(k^{s}-k^{t}\right) d\left(T^{i^{(s)}} x_{0}, T^{(s)} x_{0}, a\right) \preceq \theta .
$$

Since $k^{s}-k^{t}$ is invertible, we can obtain $d\left(T^{i^{(s)}} x_{0}, T^{(s)} x_{0}, a\right)=\theta$ by multiplying $\left(k^{s}-k^{t}\right)^{-1}$ in both sides. This means $d\left(T^{i} x_{0}, T^{j} x_{0}, a\right)=\theta$ for any $i, j, a$, which contradicts the definition of cone 2-metric spaces over Banach algebras. Hence, our claim is true. Namely, (ii) is proven.

Now, take $a=x_{0}$, for any $1 \leq i, j \leq n$, by (ii), there exists $0 \leq m \leq n$ such that

$$
d\left(T^{i} x_{0}, T^{j} x_{0}, x_{0}\right) \preceq k d\left(x_{0}, T^{m} x_{0}, x_{0}\right)=\theta,
$$

which gives (iii).

According to (ii) and (iii), for fixed $n \in \mathbb{N}$ and for any $1 \leq i, j \leq n$, there exists $0 \leq$ $j^{(1)}, j^{(2)}, \ldots, j^{(t)} \leq n$ such that

$$
\begin{aligned}
d\left(T^{i} x_{0}, T^{j} x_{0}, a\right) \preceq & k d\left(x_{0}, T^{j^{(1)}} x_{0}, a\right) \\
\preceq & k\left(d\left(T x_{0}, T^{j^{(1)}} x_{0}, a\right)+d\left(x_{0}, T x_{0}, a\right)+d\left(x_{0}, T^{j^{(1)}} x_{0}, T x_{0}\right)\right) \\
= & k\left(d\left(T x_{0}, T^{j^{(1)}} x_{0}, a\right)+d\left(x_{0}, T x_{0}, a\right)\right) \\
\preceq & k^{2} d\left(x_{0}, T^{j^{(2)}} x_{0}, a\right)+k d\left(x_{0}, T x_{0}, a\right) \\
\preceq & k^{2}\left(d\left(T x_{0}, T^{j^{(2)}} x_{0}, a\right)+d\left(x_{0}, T x_{0}, a\right)+d\left(x_{0}, T^{j^{(2)}} x_{0}, T x_{0}\right)\right) \\
& +k d\left(x_{0}, T x_{0}, a\right) \\
= & k^{2} d\left(T x_{0}, T^{j^{(2)}} x_{0}, a\right)+\left(k^{2}+k\right) d\left(x_{0}, T x_{0}, a\right) \\
\preceq & k^{3} d\left(x_{0}, T^{j^{(3)}} x_{0}, a\right)+\left(k^{2}+k\right) d\left(x_{0}, T x_{0}, a\right) \\
& \vdots \\
\preceq & k^{t} d\left(x_{0}, T^{j^{(t)}} x_{0}, a\right)+\left(k^{t-1}+\cdots+k\right) d\left(x_{0}, T x_{0}, a\right)
\end{aligned}
$$

There exists $1 \leq s<t$ such that $j^{(s)}=j^{(t)}$, then

$$
\begin{aligned}
& k^{s} d\left(x_{0}, T^{j^{(s)}} x_{0}, a\right)+\left(k^{s-1}+\cdots+k\right) d\left(x_{0}, T x_{0}, a\right) \\
& \quad \leq k^{t} d\left(x_{0}, T^{j(t)} x_{0}, a\right)+\left(k^{t-1}+\cdots+k\right) d\left(x_{0}, T x_{0}, a\right),
\end{aligned}
$$

which implies

$$
\left(k^{s}-k^{t}\right) d\left(x_{0}, T^{j^{(s)}} x_{0}, a\right) \preceq\left(k^{t-1}+\cdots+k^{s}\right) d\left(x_{0}, T x_{0}, a\right) .
$$

Hence,

$$
d\left(T^{i} x_{0}, T^{j} x_{0}, a\right) \preceq k^{s}\left(k^{s}-k^{t}\right)^{-1}\left(k^{t-1}+\cdots+k^{s}\right) d\left(x_{0}, T x_{0}, a\right)+\left(k^{s-1}+\cdots+k\right) d\left(x_{0}, T x_{0}, a\right) .
$$


On the other hand, by Lemma 3.1, we have

$$
\begin{aligned}
& k^{s}\left(k^{s}-k^{t}\right)^{-1}=k^{s}\left(\left(e-k^{t-s}\right) k^{s}\right)^{-1}=\left(e-k^{t-s}\right)^{-1}=\sum_{i=0}^{\infty}\left(k^{t-s}\right)^{i} \preceq \sum_{i=0}^{\infty} k^{i}=(e-k)^{-1}, \\
& k^{t-1}+\cdots+k^{s}=k^{s}\left(e+k+\cdots+k^{t-s-1}\right) \preceq k^{s}(e-k)^{-1} \preceq(e-k)^{-1} .
\end{aligned}
$$

Also, we can get $\left(k^{s-1}+\cdots+k\right) \preceq(e-k)^{-1}$ in a similar way. So we have

$$
d\left(T^{i} x_{0}, T^{j} x_{0}, a\right) \preceq\left((e-k)^{-1}(e-k)^{-1}+(e-k)^{-1}\right) d\left(x_{0}, T x_{0}, a\right) .
$$

In fact, we can obtain $d\left(x_{0}, T^{j} x_{0}, a\right) \preceq\left((e-k)^{-1}(e-k)^{-1}+(e-k)^{-1}\right) d\left(x_{0}, T x_{0}, a\right)$ for any $1 \leq j \leq n$ by using the same approach. Then (iv) is given.

Theorem 3.2 Let $(X, d)$ be a complete cone 2-metric space over the Banach algebra $\mathcal{A}$ and $P$ be the underlying solid cone.

(i) Suppose that $x$ is invertible and that $x^{-1} \succ \theta$ implies $x>\theta$.

(ii) Assume the mapping $T: X \rightarrow X$ satisfies

$$
d(T x, T y, a) \preceq k \bigvee\{d(x, y, a), d(x, T x, a), d(y, T y, a), d(x, T y, a), d(y, T x, a)\}
$$

for an invertible $k \in P$ with $r(k)<1$.

Then $T$ has a unique fixed point $x$ in $X$ and for every $x_{0} \in X$, the iterative sequence $\left\{T^{n} x_{0}\right\}$ converges to $x$. Besides, the following error estimate formula holds for any $c \gg \theta$ :

$$
d\left(T^{n} x_{0}, x, a\right) \preceq k^{n}\left((e-k)^{-1}(e-k)^{-1}+(e-k)^{-1}\right) d\left(x_{0}, T x_{0}, a\right)+c .
$$

Proof Take $x_{0} \in X$. Set $x_{n}=T^{n} x_{0}, i=1,2, \ldots$ For any $n, m, n<m$, it follows from Lemma 3.3 and (3.3) that

$$
\begin{aligned}
d\left(x_{n}, x_{m}, a\right)= & d\left(T x_{n-1}, T^{m-n+1} x_{n-1}, a\right) \\
\leq & k \bigvee_{0 \leq i, j \leq m-n+1}\left\{d\left(T^{i} x_{n-1}, T^{j} x_{n-1}, a\right)\right\} \\
= & k \bigvee_{n-1 \leq i, j \leq m}\left\{d\left(T^{i} x_{0}, T^{j} x_{0}, a\right)\right\} \\
\leq & k^{2} \bigvee_{n-2 \leq i, j \leq m}\left\{d\left(T^{i} x_{0}, T^{j} x_{0}, a\right)\right\} \\
& \vdots \\
\preceq & k^{n} \bigvee_{0 \leq i, j \leq m}\left\{d\left(T^{i} x_{0}, T^{j} x_{0}, a\right)\right\} \\
\preceq & k^{n}\left((e-k)^{-1}(e-k)^{-1}+(e-k)^{-1}\right) d\left(x_{0}, T x_{0}, a\right) .
\end{aligned}
$$

Hence, by Lemma 2.5 and the fact that $\left\|k^{n}\left((e-k)^{-1}(e-k)^{-1}+(e-k)^{-1}\right) d\left(x_{0}, T x_{0}, a\right)\right\| \rightarrow 0$ $(n \rightarrow \infty)$, we see that, for any $c \in \mathcal{A}$ with $\theta \ll c$, there exists $N \in \mathbb{N}$ such that for any 
$m>n>N$, we have

$$
d\left(x_{n}, x_{m}, a\right) \preceq k^{n}\left((e-k)^{-1}(e-k)^{-1}+(e-k)^{-1}\right) d\left(x_{0}, T x_{0}, a\right) \ll c .
$$

So $\left\{x_{n}\right\}$ is a Cauchy sequence in $X$. As $X$ is complete, there exists $x \in X$ such that $x_{n} \rightarrow x$ $(n \rightarrow \infty)$. Let $m \rightarrow \infty$ and by Lemma 3.2, we have

$$
d\left(x_{n}, x, a\right) \preceq d\left(x_{n}, x_{m}, a\right)+c \preceq k^{n}\left((e-k)^{-1}(e-k)^{-1}+(e-k)^{-1}\right) d\left(x_{0}, T x_{0}, a\right)+c
$$

for all $n \geq 1$ and any $c \gg \theta$, which gives the error estimate formula.

For all $a \in X$, we have

$$
d\left(x_{n+1}, T x, a\right) \preceq k \bigvee\left\{d\left(x_{n}, x, a\right), d\left(x_{n}, x_{n+1}, a\right), d(x, T x, a), d\left(x_{n+1}, x, a\right), d\left(x_{n}, T x, x\right)\right\}
$$

By Proposition 3.2, for any $\theta \ll c$ there exists $N \in \mathbb{N}$ such that for all $n>N$,

$$
\begin{array}{ll}
k d\left(x_{n}, x, a\right) \ll \frac{c}{2}, & k d\left(x_{n}, x_{n+1}, a\right) \ll \frac{c}{2}, \\
k d\left(x_{n+1}, x, a\right) \ll \frac{c}{2}, & k d\left(x_{n}, T x, x\right) \ll \frac{c}{2} .
\end{array}
$$

Hence, we have $d\left(x_{n+1}, T x, a\right) \ll \frac{c}{2}$ or $d\left(x_{n+1}, T x, a\right) \preceq k d(x, T x, a)$ for all $n>N$. By Lemma 3.2, it is easy to see that $d(x, T x, a) \ll c$ or $(e-k) d(x, T x, a) \ll c$, which together with Proposition 3.2 implies that $d(x, T x, a)=\theta$ for any $a \in X$ as $e-k$ is invertible. Furthermore, $T x=x$.

Suppose that $y$ is another fixed point of $T$ in $X$, then

$$
d(x, y, a) \preceq k \bigvee\{d(x, y, a), d(x, x, a), d(y, y, a), d(x, y, a), d(y, x, a)\},
$$

which gives $d(x, y, a) \preceq \theta$ or $d(x, y, a) \preceq k d(x, y, a)$. So $d(x, y, a)=\theta$. Then $x=y$.

Remark 3.2 If we replace the Banach algebra $\mathcal{A}$ with the real numbers field $\mathbb{R}$ in Theorem 3.2, then we get Theorem 1.2, so Theorem 1.2 is just a corollary of Theorem 3.2. Moreover, it should be noted that, in a cone 2-metric space over the Banach algebra $\mathcal{A}$, for any pair of given elements, they may not be comparable, which is the key difficulty for us in proving Theorem 3.2. Fortunately, we found an effective way for it, and our way may be helpful for other researchers to deal with similar problems.

We conclude the paper with an example, which can illustrate the result of Theorem 3.1.

Example 3.1 Let $\mathcal{A}=\mathbb{R}^{2}$. For each $\left(x_{1}, x_{2}\right) \in \mathcal{A},\left\|\left(x_{1}, x_{2}\right)\right\|=\left|x_{1}\right|+\left|x_{2}\right|$. The multiplication is defined by $x y=\left(x_{1}, x_{2}\right)\left(y_{1}, y_{2}\right)=\left(x_{1} y_{1}, x_{1} y_{2}+x_{2} y_{1}\right)$. Then $\mathcal{A}$ is a Banach algebra with unit $e=(1,0)$. Let $P=\left\{\left(x_{1}, x_{2}\right) \in \mathbb{R}^{2} \mid x_{1}, x_{2} \geq 0\right\}$. Then $P$ is a cone in $\mathcal{A}$.

Let $X=\left\{(x, 0) \in \mathbb{R}^{2} \mid 0 \leq x \leq 1\right\} \cup\left\{(0, x) \in \mathbb{R}^{2} \mid 0 \leq x \leq 1\right\}$. The mapping is defined by $d\left(\alpha_{1}, \alpha_{2}, \alpha_{3}\right)=d_{1}\left(\beta_{1}, \beta_{2}\right)$ where $\alpha_{1}, \alpha_{2}, \alpha_{3} \in X$ and $\beta_{1}, \beta_{2} \in\left\{\alpha_{1}, \alpha_{2}, \alpha_{3}\right\}$ are such that $\| \beta_{1}-$ $\beta_{2} \|=\min \left\{\left\|\alpha_{1}-\alpha_{2}\right\|,\left\|\alpha_{2}-\alpha_{3}\right\|,\left\|\alpha_{3}-\alpha_{2}\right\|\right\}$ and

$$
d_{1}((x, 0),(y, 0))=\left(\frac{5}{4}|x-y|,|x-y|\right)
$$




$$
\begin{aligned}
& d_{1}((0, x),(0, y))=\left(|x-y|, \frac{3}{4}|x-y|\right) \\
& d_{1}((x, 0),(0, y))=d_{1}((0, y),(x, 0))=\left(\frac{5}{4} x+y, x+\frac{3}{4} y\right) .
\end{aligned}
$$

Then $(X, d)$ is a complete cone 2-metric space over the Banach algebra $\mathcal{A}$.

Now we define mappings $T_{i}: X \rightarrow X(i \geq 1)$ by

$$
T_{i}((x, 0))=\left(0,\left(\frac{1}{12}\right)^{\frac{1}{2 i-1}}\left(\frac{3}{2}\right)^{\frac{i-1}{2 i-1}} x\right)
$$

and

$$
T_{i}((0, x))=\left(\left(\frac{1}{12}\right)^{\frac{1}{2 i-1}}\left(\frac{3}{2}\right)^{\frac{-i}{2 i-1}} x, 0\right) .
$$

Then it is not difficult to verify that $T_{i}^{2 i-1}((x, 0))=\left(0, \frac{1}{12} x\right)$ and $T_{i}^{2 i-1}((0, x))=\left(\frac{1}{18} x, 0\right)$. Hence, it follows from [15] that $T_{i}$ satisfies the contractive condition

$$
d\left(T_{i}^{m_{i}} x, T_{j}^{m_{j}} y, a\right) \preceq k_{1} d\left(x, T_{i}^{m_{i}} x, a\right)+k_{2} d\left(y, T_{j}^{m_{j}} y, a\right)+k_{3} d(x, y, a)
$$

for all $x, y, a \in X$ and $i \geq 1$, where $m_{i}=2 i-1, k_{1}=\left(\frac{1}{3}, 0\right), k_{2}=\left(\frac{1}{3}, 0\right), k_{3}=\left(\frac{1}{4}, 0\right)$. Besides, $r\left(k_{1}\right)=r\left(k_{2}\right)=\frac{1}{3}, r\left(k_{3}\right)=\frac{1}{4}$. By Theorem 3.1, we see that $T_{i}$ has a unique fixed point $(0,0)$ for all $i \geq 1$.

\section{Competing interests}

The authors declare that they have no competing interests.

\section{Authors' contributions}

All authors contributed equally to the writing of this paper. All authors read and approved the final manuscript.

\section{Acknowledgements}

The authors are grateful to the referees and the editors for valuable comments and suggestions, which have greatly improved the original manuscript.

Received: 18 May 2015 Accepted: 5 November 2015 Published online: 14 November 2015

\section{References}

1. Gähler, S: 2-metrische Räume und ihre topologische strukturen. Math. Nachr. 26, 115-148 (1963)

2. Gähler, S: Über die Uniformisierbarkeit 2-metricsche Raume. Math. Nachr. 28, 235-244 (1965)

3. Gähler, S: Zur geometric 2-metrische Räume. Rev. Roum. Math. Pures Appl. 11, 665-667 (1966)

4. Sharma, P, Sharma, B, Iseki, K: Contractive type mapping in 2-metric space. Math. Jpn. 21, 67-70 (1976)

5. Rhoades, B: Contractive type mapping on a 2-metric space. Math. Nachr. 91, 151-155 (1979)

6. Huang, L, Zhang, X: Cone metric spaces and fixed point theorems of contractive mappings. J. Math. Anal. Appl. 332 1468-1476 (2007)

7. Kadelburg, Z, Radenović, S: Some common fixed point results in non-normal cone metric spaces. J. Nonlinear Sci. Appl. 3(3), 193-202 (2010)

8. Kadelburg, Z, Murthy, P, Radenović, S: Common fixed points for expansive mappings in cone metric spaces. Int. J. Math. Anal. 5(27), 1309-1319 (2011)

9. Radenović, S, Radojević, S, Pantelić, S, Pavlović, M: Ćirić type theorems in abstract metric spaces. Theor. Math. Appl. 2(1), 89-102 (2012)

10. Kadelburg, Z, Radenović, S: A note on various types of cones and fixed point results in cone metric spaces. Asian J. Math. Appl. 2013, Article ID ama0104 (2013)

11. Gajić, L, Rakočević, V: Quasi-contractions on a nonnormal cone metric space. Funct. Anal. Appl. 46(1), 75-79 (2012)

12. Liu, H, Xu, S: Cone metric spaces with Banach algebras and fixed point theorems of generalized Lipschitz mappings. Fixed Point Theory Appl. 2013, 320 (2013)

13. Huang, H, Radenović, S, Došenović, T: Some common fixed point theorems on c-distance in cone metric spaces over Banach algebras. Appl. Comput. Math. 14(2), 180-193 (2015)

14. Huang, H, Radenović, S: Common fixed point theorems of generalized Lipschitz mappings in cone $b$-metric spaces over Banach algebras and applications. J. Nonlinear Sci. Appl. 8, 787-799 (2015) 
15. Singh, B, Jain, S, Bhagat, P: Cone 2-metric space and fixed point theorem of contractive mappings. Comment. Math. 52(2), 143-151 (2012)

16. Rudin, W: Functional Analysis, 2nd edn. McGraw-Hill, New York (1991)

17. Xu, S, Radenović, S: Fixed point theorems of generalized Lipschitz mappings on cone metric spaces over Banach algebras without assumption of normality. Fixed Point Theory Appl. 2014, 102 (2014)

18. Radenović, S, Rhoades, B: Fixed point theorem for two non-self mappings in cone metric spaces. Comput. Math. Appl. 57, 1701-1707 (2009)

19. Dordević, M, Dorić, D, Kadelburg, Z, Radenović, S, Spasić, D: Fixed point results under c-distance in tvs-cone metric spaces. Fixed Point Theory Appl. 2011, 29 (2011). doi:10.1186/1687-1812-2011-29

20. Kadelburg, Z, Radenović, S: A note on various types of cones and fixed point results in cone metric spaces. Asian J. Math. Appl. 2013, Article ID ama0104 (2013)

Submit your manuscript to a SpringerOpen ${ }^{\circ}$ journal and benefit from:

- Convenient online submission

Rigorous peer review

- Immediate publication on acceptance

- Open access: articles freely available online

- High visibility within the field

- Retaining the copyright to your article 